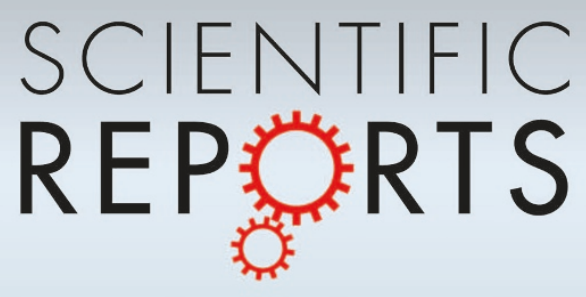

OPEN

SUBJECT AREAS:

TRANSFORMATION

OPTICS

ELECTRICAL AND ELECTRONIC ENGINEERING

METAMATERIALS

APPLIED MATHEMATICS

Received

26 April 2013

Accepted

21 May 2013

Published

24 June 2013

Correspondence and requests for materials should be addressed to A.J.D. (adanner@nus. edu.sg)

\section{Exploiting design freedom in biaxial dielectrics to enable spatially overlapping optical instruments}

\author{
Alireza Akbarzadeh, Cheng-Wei Qiu \& Aaron J. Danner
}

Department of Electrical and Computer Engineering, National University of Singapore, Singapore 117576.

The optical behavior of gradient biaxial dielectrics has not been widely explored in the literature due to their complicated nature, but the extra degrees of freedom in the index tensor have the potential of yielding useful optical instruments which are otherwise unachievable. In this work, a design method is described in detail which allows one to combine the behavior of up to four totally independent isotropic optical instruments in an overlapping region of space. This is non-trivial because of the mixing of the index tensor elements in the Hamiltonians; previously known methods only handled uniaxial dielectrics (where only two independent isotropic optical functions could overlap). The biaxial method introduced also allows three-dimensional multi-faced Janus devices to be designed; these are worked out in an example of what is possible to design with the method.

$\mathrm{t}$ is remarkable in modern optics that there is still no general method of designing a gradient index device when presented with a desired optical behavior and the correct form of the Hamiltonian, even in the isotropic case. However, much can be learned from the case of spherical symmetry, where general design methods do exist, both for isotropic and uniaxial cases ${ }^{1,2}$. Spherically symmetric lenses are also of wide interest because of their unique ability to work equally well in all directions, and it stands to reason that there exist a number of spherically symmetric lenses that are "named" because of their great utility, viz. Maxwell's Fisheye ${ }^{3}$, the Eaton Lens ${ }^{4}$, the Luneberg Lens ${ }^{5}$, the Invisible Sphere ${ }^{1,2}$, the Pendry Cloak ${ }^{6}$, and the Miñano Lens ${ }^{7}$, to name just a few. Spherical symmetry is also important because ray Hamiltonians have the same form in all orthonormal coordinate systems $^{8,9}$, so results in Hamiltonian optics in this system can often be generalized to other coordinate systems. In this work, we explore the design of gradient-permittivity biaxial dielectrics in this special system, which is much more complicated than it may first seem: although the permittivity tensor contains only three diagonal elements, they are not separable in the Hamiltonians that determine the ray trajectories ${ }^{8}$-light traveling through a biaxial device will generally be affected by all three gradient index tensors, resulting in long and complicated Hamiltonians. This occurs because light polarization can be continuously rotated in such a device. Though complex, biaxial optical instruments represent a potentially important way to achieve certain kinds of functionality in optics that would otherwise be impossible. In this work, for example, it will be shown that one can take any four independently-chosen spherically-symmetric lenses and combine their optical functions in an overlapping spherical region of space with a single biaxial lens, with each function assigned to a specific polarization and set of planes. We define "optical function" here to include the behavior of any possible lens or optical instrument that can be designed with an isotropic permittivity. (Note that this is extremely general, since it encompasses anything that can be defined by a spatially-dependent refractive index profile.) Due to the mixing of the tensor elements, it will also be shown that four is the maximum number of independent isotropic lens functions that can be combined. If more lens functions were desired in an overlapping region of space, symmetry would be broken.

A successful attempt to combine two optical functions into a single device with uniaxial symmetry was previously shown in $^{2}$ and such a device was realized experimentally in $^{10}$. The work of ${ }^{2}$ was also general: it combined arbitrary lens functions in overlapping physical space (where light entering an arbitrary point of a lens may interact with the entire body of the lens, for all points on the lens) and it is this generality that made the finding significant. $\mathrm{In}^{2}$, through a regressive formulation on the basis of transformation optics, a new degree of freedom was induced into a dielectric uniaxial medium to make two polarizations do two different tasks simultaneously, while maintaining spherical symmetry. Lenses could be rotated arbitrarily without altering light trajectories at all. But unfortunately the integration over the physical extent of the device to determine the 
transformation function in ${ }^{2}$ is not always convergent. And even in the converging cases, that method can provide only one transformation function for each pair of functionalities which means that method remains somewhat limited from an experimental point of view.

The design method to be introduced here relaxes that constraint somewhat, and can be readily applied to improve existing designs in metamaterials. For example, devices designed with transformation optics ${ }^{1,6,11}$ require a nonunity magnetic permeability resulting in either loss or sacrifice of device functionality for one polarization ${ }^{2,12-15}$, but this polarization can be "recovered" with the biaxial method to take on new optical functions. Biaxial dielectrics could potentially be fabricated by creating composite materials (asymmetric air inclusions in glass, for example) that have practically no loss or dispersion at optical wavelengths. The field of dielectric device design, with no need for metals or chirality, remains rich for exploration.

Another application of the biaxial method is to build upon interesting existing work in the area of "Janus" devices", where twodimensional dielectric devices having two different optical functions in two different directions can be made. The authors of ${ }^{16}$ employed transformation optics in two vertical directions independently and by invoking a quasi-conformal mapping technique ${ }^{11}$, they achieved a nearly isotropic permittivity profile for their Janus device. However, the designed metadevice, like almost all transformation optics devices, was limited to only one polarization and furthermore is restricted to only a few permissible optical functions. Here, we extend this work to three dimensions and encompass both polarizations.

In this report, we summarize the biaxial method and then describe it with two design examples. We first find and then factor the Hamiltonian into two terms which are responsible for trajectories of light rays in a plane of interest in a spherical system. Taking advantage of the factorization, we examine the light rays in two orthogonal planes with respect to all possible polarizations, and relate the desired optical functions to the (non-independent) permittivity tensor elements through careful spatial sorting of the rays by polarization. The first design example is to combine four isotropic lenses into one biaxial lens. We combine these lenses: an Eaton Lens, a 135-degree universal ray rotator, a 90-degree universal ray rotator, and an Invisible Sphere. Any lenses could have been chosen. The second design example is to create a three dimensional polarizationindependent two-faced Janus device comprising an Eaton lens at one face and an Invisible Sphere at the other. It should be noted that all of our chosen lenses to be combined contain singularities in their original index profiles (they were chosen for simplicity); hence, the resulting permittivity tensor will also contain singularities.

\section{Results}

We start with a standard spherical coordinate system. In a sphere the principle planes are the equatorial and polar planes. The latitudinal plane which slices the sphere into northern and southern hemispheres is the equatorial plane and the longitudinal planes which cross the north and south poles are called the polar planes. These two plane sets and the basis vectors at their boundaries are shown in Figure 1. It should be noted that in any sphere, we have a unique equatorial plane and infinite number of polar planes. It is along these two orthogonal sets of planes where, for each of two polarizations, we will prescribe lens functions. Based on the factorization of the Hamiltonian of the biaxial dielectric media, one can control the ray trajectories for each polarization (in-plane and out-of-plane) with respect to an orthogonal plane set of the meta-device; the set of principal planes is a convenient choice. Before describing the design details of the design process, it is worth understanding the two example results.

The first designed spherical meta-device is presented in Figure 2. Note that although two spheres are shown in Figures 2(c) and 2(d), respectively, it is just a single device with rays for different polarizations
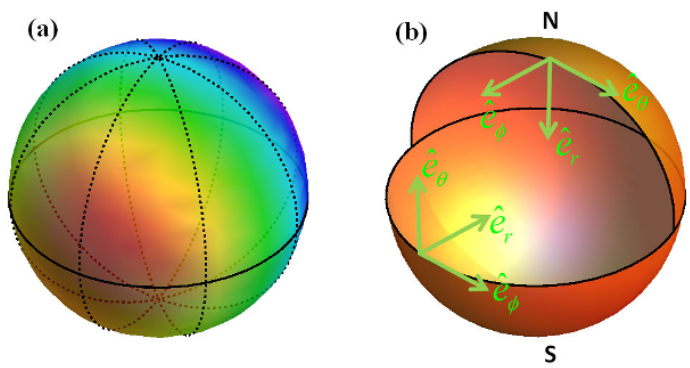

Figure $1 \mid$ (a) Illustration of equatorial and polar planes in a sphere (the solid circle is the equatorial plane and the dashed circles are polar planes); (b) alignments of basis vectors along equatorial and polar planes.

illustrated. The sphere (radius $R=1$ ) has three concentric layers of radial thicknesses $0.51,0.34$ and 0.15 from the innermost to the outermost layer, respectively. As seen in Figure 2(c), for the in-plane polarizations all incoming rays are sorted into the middle layer and are bent by 90 degrees or 180 degrees within this layer in the equatorial or polar planes, respectively. For out-of-plane polarizations in Figure 2(d), all incoming rays are sorted into the innermost layer and are deflected by 360 or 135 degrees along equatorial or polar planes, respectively. In aggregate we have four different lens functions implemented simultaneously in orthogonal planes comprising the entire sphere. The design method is complicated by the necessary sorting of rays by polarization, and ensuring that correct sorting is maintained for all planes.

Of course, the example just described is polarization-sensitive by design. If we would instead like to obtain a two-faced Janus device that works for unpolarized light, it is just necessary to equate the inplane and the out-of-plane polarization functionalities along each of the equatorial and polar planes. Shown in Figure 3(c) are the ray paths for such a Janus device along the equatorial plane, where each incoming ray splits into two polarizations at $R=0.85$, travel in different paths along the plane, recombine again, and exit with 360 degrees deflection. This splitting and recombining is necessary to make the device work for arbitrary functions along the polar planes: Figure $3(\mathrm{~d})$ shows that in a polar plane, each unpolarized ray splits into in-plane and out-of-plane polarized rays, recombines, and achieves 180 degrees deflection. It should be noted that as the profiles of the device are radially symmetric, the in-plane and out-of-plane polarized rays are confined to their own equatorial and polar planes ${ }^{1}$. The obtained profile indices for this Janus device are given in Figure 3(a) and Figure 3(b). We now describe how such lenses can be designed in general.

\section{Discussion}

It is known that in general the permittivity tensor $\varepsilon$ of a reciprocal medium is symmetric and therefore we can always find a specific orthonormal coordinate system (i.e. principle coordinate system) in which the permittivity tensor can be written in the form of a diagonal matrix. So in the principle coordinate system $\left\{x_{1}, x_{2}, x_{3}\right\}$, for a dielectric (i.e. permeability equal to one, $\mu=1$ ) biaxial medium we have

$$
\varepsilon=\operatorname{diag}\left\{n_{1}^{2}, n_{2}^{2}, n_{3}^{2}\right\}
$$

where $n_{1}, n_{2}$ and $n_{3}$ are the refractive indices along the principle axes and diag refers to the diagonal tensor elements. According to the generalized method presented in ${ }^{9}$, the Hamiltonian of a biaxial medium in the principle coordinate system is of the form

$$
\begin{aligned}
\mathcal{H}= & k_{1}^{4} n_{1}^{2}+\left(k_{2}^{2}+k_{3}^{2}-n_{1}^{2}\right)\left(k_{3}^{2} n_{3}^{2}+\left(k_{2}^{2}-n_{3}^{2}\right) n_{2}^{2}\right)+ \\
& k_{1}^{2}\left(k_{3}^{2}\left(n_{3}^{2}-n_{1}^{2}\right)-n_{1}^{2}\left(n_{3}^{2}+n_{2}^{2}\right)+k_{2}^{2}\left(n_{1}^{2}+n_{2}^{2}\right)\right)
\end{aligned}
$$


(a)
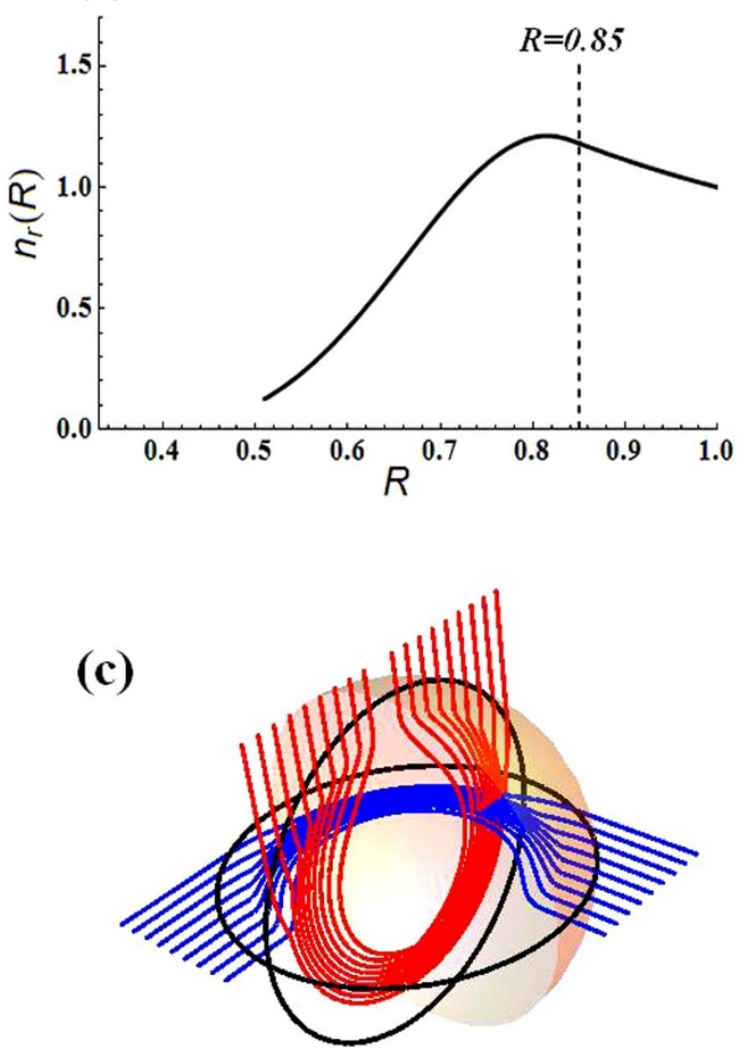

(b)

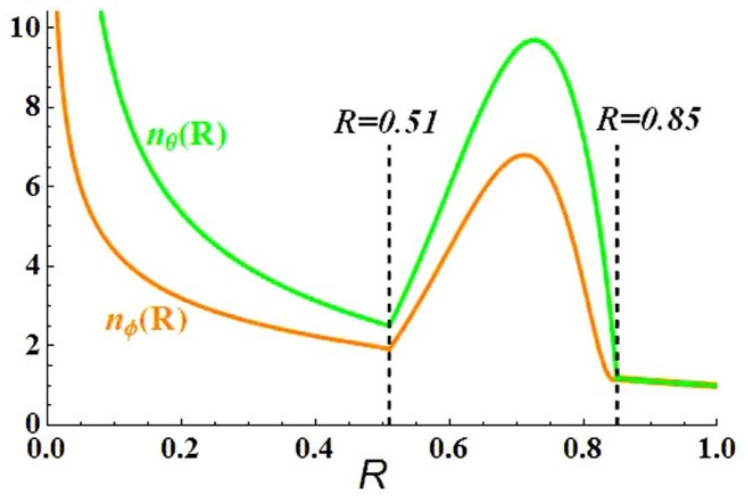

(d)

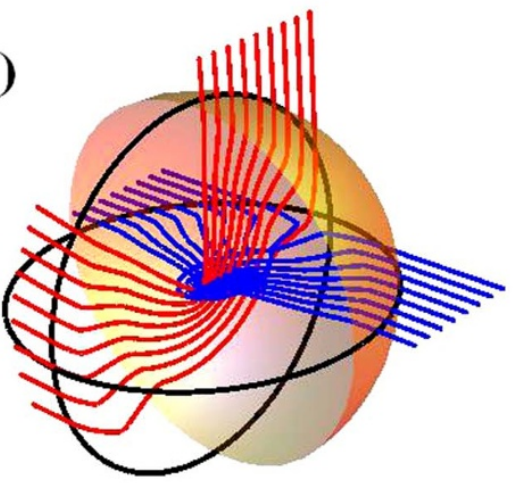

Figure $2 \mid$ (a,b) Index profiles for $n_{r}, n_{\theta}$ and $n_{\phi}$. The middle and the inner layer radii are 0.85 and 0.51 , respectively. The profile $n_{r}$ within the inner layer is undefined, as it has no role in the shown functionalities. (c) The performance of the device for the in-plane polarization along polar (red rays) and equatorial (blue rays) planes. (d) The performance of the device for the out-of-plane polarization along polar (red rays) and equatorial (blue rays) planes.

where $k_{i}(i=1,2,3)$ are the components of the wave vector. The form of Equation (2) already illustrates why it is difficult to design devices in biaxial dielectrics - the ray trajectories essentially depend on all three indices simultaneously. One could not design a biaxial device by independently choosing the three indices, for example, as the resulting sphere would give unpredictable ray trajectories. The Hamiltonian in a biaxial medium is of degree four for each $k_{i}$ and hence in the wave vector domain it constructs a special surface which intersects each axis at four conjugate points. This surface, which is sometimes called the wave surface or optical indicatrix ${ }^{8}$, is the combination of a sphere and an oval. Due to the structure of the wave surface, an incident ray splits into two refracting rays at the interface of a biaxial medium (double refraction), though in some instances they may overlap or become evanescent. It should be recalled that, as discovered by Hamilton ${ }^{17}$ at the diabolical points ${ }^{18}$, where there is a degeneracy in the direction of the normal to the Hamiltonian surface, conical refraction occurs and a cone of rays appears within the medium. However, we can factor the Hamiltonian into two terms which have nontrivial roots. Following the method of Born and $\mathrm{Wolf}^{8}$ with detailed algebraic steps provided in the Supplementary Information, we can express the Hamiltonian as

$$
\mathcal{H}=\mathcal{H}_{a} \mathcal{H}_{b} \mathcal{H}_{c}
$$

where for $\mathcal{H}_{a}, \mathcal{H}_{b}$ and $\mathcal{H}_{c}$ we have

$$
\mathcal{H}_{a}=\frac{k_{1}^{2} n_{1}^{2}+k_{2}^{2} n_{2}^{2}+k_{3}^{2} n_{3}^{2}}{k^{2}}
$$

$$
\begin{aligned}
\mathcal{H}_{b, c}= & -k^{2}+n_{2}^{2}+\frac{1}{2\left(k_{1}^{2} n_{1}^{2}+k_{2}^{2} n_{2}^{2}+k_{3}^{2} n_{3}^{2}\right)} \\
& \left\{\left(k_{1}^{2} n_{1}^{2}+k_{2}^{2} n_{2}^{2}\right)\left(n_{2}^{2}-n_{3}^{2}\right)-\right. \\
& \left(k_{2}^{2} n_{2}^{2}+k_{2}^{2} n_{2}^{2}\right)\left(n_{1}^{2}-n_{2}^{2}\right) \pm\left[k_{1}^{4} n_{1}^{4}\left(n_{2}^{2}-n_{3}^{2}\right)^{2}+\right. \\
& \left(k_{3}^{2} n_{3}^{2}\left(n_{1}^{2}-n_{2}^{2}\right)+k_{2}^{2} n_{2}^{2}\left(n_{1}^{2}-n_{3}^{2}\right)\right)^{2}+2 k_{1}^{2} n_{1}^{2}\left(n_{2}^{2}-n_{3}^{2}\right) \\
& \left.\left.\left(k_{3}^{2} n_{3}^{2}\left(n_{2}^{2}-n_{1}^{2}\right)+k_{2}^{2} n_{2}^{2}\left(n_{1}^{2}-n_{3}^{2}\right)\right)\right]^{0.5}\right\}
\end{aligned}
$$

In equation (5), the positive branch is for $\mathcal{H}_{b}$ and the negative is for $\mathcal{H}_{c}$, where $k^{2}=k_{1}^{2}+k_{2}^{2}+k_{3}^{2}$. At diabolical points, we have $\mathcal{H}_{b}=$ $\mathcal{H}_{c}=0$ and the ray trajectories cannot be defined. Essentially the Hamiltonian surface is now divided into outer and inner shells. The features of these two shells as well as the whole Hamiltonian surface are depicted in Figure $2 \mathrm{~S}$ of the Supplementary Information.

In the factored form, the Hamiltonian is broken into two terms $\mathcal{H}_{b}$ and $\mathcal{H}_{c}$ which individually describe either of the two orthogonally-polarized rays along a plane of interest and therefore by manipulating these two terms it is possible to control the ray trajectories. ( $\mathcal{H}_{a}$ is just a prefactor.) Unfortunately along any arbitrary plane, the factored expressions in Equation (5) are complicated. However, along principle planes (i.e. one of $k_{i}$ vanishes) of the biaxial medium the factored terms $\mathcal{H}_{b}$ and $\mathcal{H}_{c}$ turn out to be simpler, so it is convenient choice for setting the lens functions. Even along these planes, however, the expressions share many terms and in order to control the ray trajectories, we cannot simply play with the permittivity elements ad hoc to achieve our desired lens functions, as random 
(a)

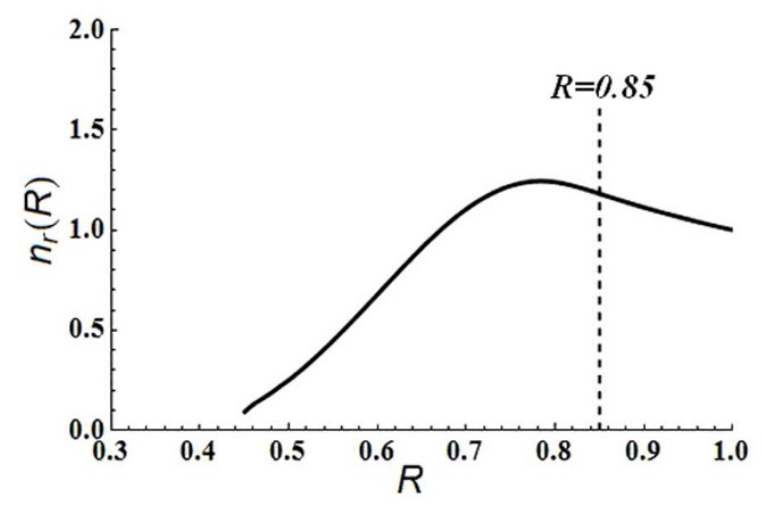

(c)

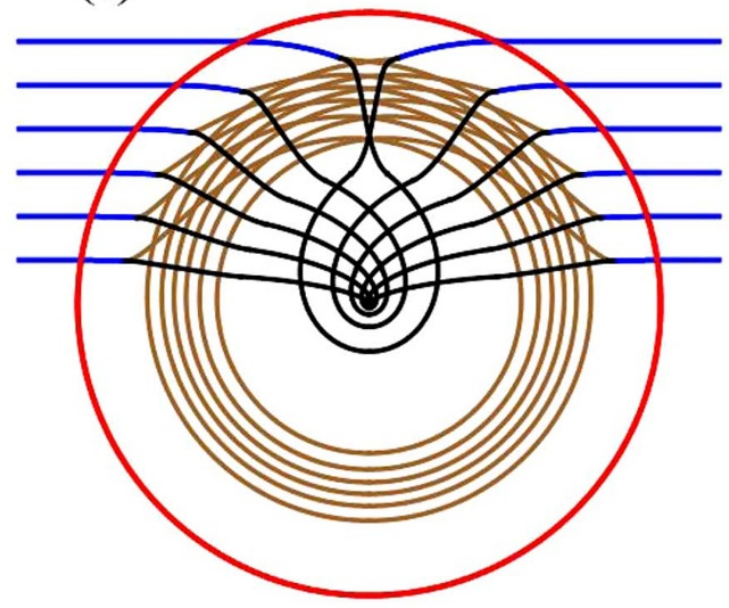

(b)

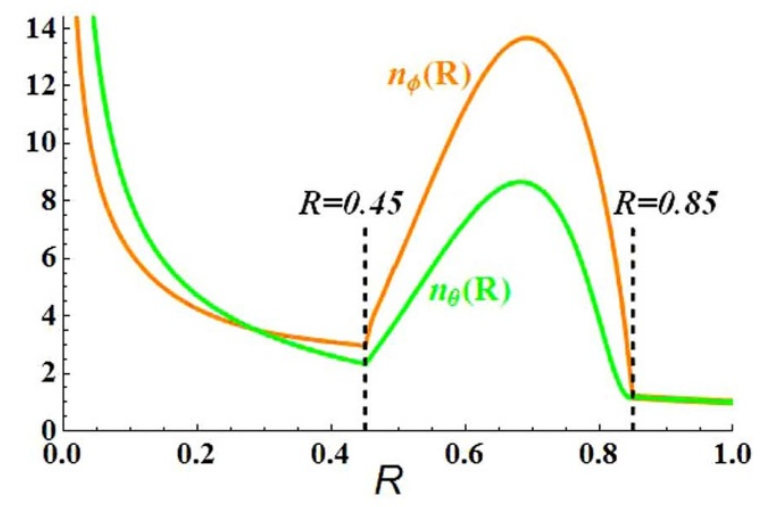

(d)

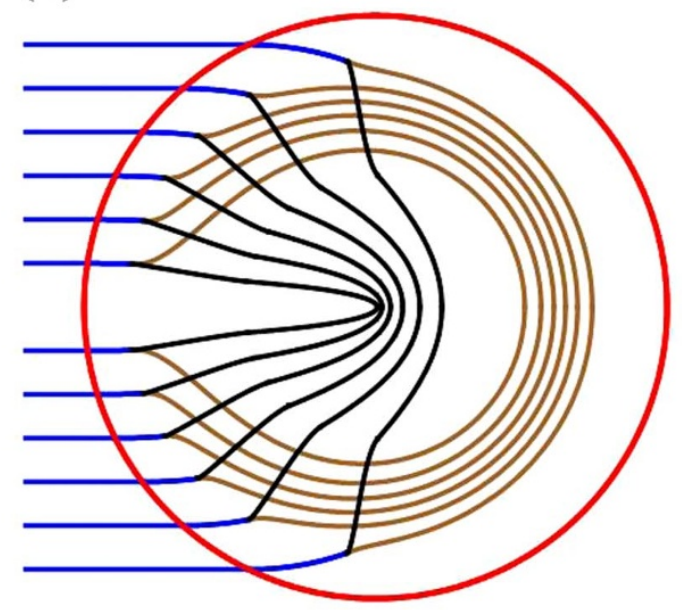

Figure $3 \mid$ (a,b) The profile indices $n_{r}, n_{\theta}$ and $n_{\phi}$ for the Janus device. In this design, the middle and the inner layer radii are 0.85 and 0.45 , respectively. (c) The ray trajectories for in-plane (brown rays) and out-of plane polarizations (black rays) along the equatorial plane. (d) The ray trajectories for in-plane (brown rays) and out-of plane polarizations (black rays) along polar planes.

guessing may not lead to interesting functions for all polarizations. To get around this problem, we make use of transformation optics to open space in the physical domain (the principle planes) for correcting the paths of the rays with the out-of-plane polarizations, while the opened space cannot be seen by the in-plane polarizations. This corresponds to the sorting-by-layer described above. Although we believe this method can work in any orthogonal coordinate geometry, we will now use the spherical coordinate system as a specific example frame to depict the validity of this method.

According to Figure 1(b) the Hamiltonian of a biaxial dielectric medium with permittivity tensor $\varepsilon=\operatorname{diag}\left\{\varepsilon_{r}=n_{r}^{2}, \varepsilon_{\theta}=n_{\theta}^{2}, \varepsilon_{\phi}=n_{\phi}^{2}\right\}$ in a spherical geometry along the equatorial and polar planes can be written as

$$
\begin{gathered}
\mathcal{H}_{\text {equatorial }}=\left(k_{r}^{2}+k_{\phi}^{2}-n_{\theta}^{2}\right)\left(k_{\phi}^{2} n_{\phi}^{2}+\left(k_{r}^{2}-n_{\phi}^{2}\right) n_{r}^{2}\right) \\
\mathcal{H}_{\text {polar }}=\left(k_{r}^{2}+k_{\theta}^{2}-n_{\phi}^{2}\right)\left(k_{\theta}^{2} n_{\theta}^{2}+\left(k_{r}^{2}-n_{\theta}^{2}\right) n_{r}^{2}\right)
\end{gathered}
$$

where $\vec{k}=\hat{e}_{r} k_{r}+\hat{e}_{\theta} k_{\theta}+\hat{e}_{\phi} k_{\phi}$ is the wave vector. It should be noted that we assume our spherical device is radially symmetric, i.e. $n_{i}=n_{i}(r)$ where $i=r, \theta, \phi$, and the sphere radius is $R=1$. The device radius could always be changed, and all the equations and parameters would be rescaled accordingly. Equation (6) indicates that along the equatorial plane, one ray (with the out-of-plane polarization) behaves like it would in an isotropic medium of index $n_{\theta}$ (the first factor), and the other ray with the in-plane polarization behaves as if it were in a complicated singly refracting medium influenced by both $n_{r}$ and $n_{\phi}$ (the second factor). For polar rays (equation (7)), it is as if there were an isotropic medium $n_{\phi}$ (the first factor) and another complicated medium of $n_{r}$ and $n_{\theta}$ (the second factor). Indeed four different functions can theoretically be achieved for equatorial and polar rays, but only through careful design because the four terms share some of the permittivity tensor elements.

\section{Methods}

Transformation optics is used to open a hole of radius $a$ at the center of the sphere to have some space to correct the ray trajectories for the out-of-plane polarizations. Now suppose for the in-plane polarizations we would like the spherical device to offer function "A" for equatorial rays and function "B" for polar rays. And also suppose that these two functions can be individually implemented via isotropic and radially symmetric index profiles. Let us take the isotropic profiles of functions A and B as $n_{e}(r)$ and $n_{p}(r)$, respectively, where these two profiles are assumed to be impedance matched with the vacuum at the boundary. Considering $n_{e}(r)$ and $n_{p}(r)$ as virtual indices, if we employ transformation functions $r_{r \phi}=r_{r \phi}(R)$ and $r_{r \theta}=r_{r \theta}(R)$, where $0 \leq r_{r \phi} \leq 1$ and $0 \leq r_{r \theta} \leq 1$ refer to virtual space along equatorial and polar planes respectively and $a \leq R \leq 1$ denotes the physical space, then after normalization we have

$$
\varepsilon=\operatorname{diag}\left\{n_{r}^{2}=n_{e}^{2}\left(\frac{r_{r \phi}}{R}\right)^{2}=n_{p}^{2}\left(\frac{r_{r \theta}}{R}\right)^{2}, n_{\theta}^{2}=n_{p}^{2}\left(\frac{d r_{r \theta}}{d R}\right)^{2}, n_{\phi}^{2}=n_{e}^{2}\left(\frac{d r_{r \phi}}{d R}\right)^{2}\right\}
$$

Similar to ${ }^{2}$, as we would like to work with dielectrics, we let $\mu=\{1,1,1\}$ in physical space. From the first expression in (8), we obtain $n_{e} r_{r \phi}=n_{p} r_{r \theta}$ leading to $d n_{e}(r) /\left.d r\right|_{r=1}=d n_{p}(r) /\left.d r\right|_{r=1}$. Not any arbitrary pair of profiles satisfy this equality. In order to overcome this obstacle, we break each of the $n_{e}(r)$ and $n_{p}(r)$ profiles into two layers; outer layers $r_{1} \leq r \leq 1$ with similar profile indices and inner layers $0 \leq r \leq r_{1}$ with different profile indices. The outer profile should be designed so that 
incoming rays all spiral into the inner layer. Any function which is equal to one at $r=$ 1 and larger than $1 / r$ in the range $r_{1} \leq r \leq 1$ can do the job for the outer layer ${ }^{2}$. In order to design the profile indices within the inner layers, we use an implicit integral equation derived from conservation of angular momentum ${ }^{19-21}$ (see Supplementary Information). Then afterwards, with careful choice of transformation functions $r_{r}$ and $r_{r \theta}$, we open a hole at the center of the inner layer into which the out-of-plane polarized rays should spiral. Since the out-of-plane rays are governed by $n_{\phi}$ and $n_{\theta}$ along polar and equatorial planes, respectively, we should have

$$
n_{\phi}(R), n_{\theta}(R)>\frac{1}{R} \quad \text { for } a<R<r_{1}
$$

Then considering two other arbitrary functions like " $\mathrm{C}$ " and " $\mathrm{D}$ " for the out-ofplane polarized rays along equatorial and polar planes, we can calculate the required turning angles for each function and resort to the implicit integral equation method again to find $n_{\theta}(R)$ and $n_{\phi}(R)$ in the region $0<R \leq a$. It should be said that $n_{r}$ in the range $0<R \leq a$ remains unchosen and can be any well-defined mathematical function, as it plays no role in the functionalities of the device along equatorial and polar planes. It also turns out that one cannot design arbitrary functions for other planes through the sphere, as they will influence choices already made for the index profiles in a dependent way. Additional explanation is in the Supplementary Information.

In conclusion, the general Hamiltonian for biaxial dielectric media was factored into two terms. On the basis of this factorization and with the use of transformation optics, a method of designing a radially symmetric biaxial dielectric device was described which allows one to simultaneously combine four isotropic lens functions in an overlapping spherical region. This is the greatest number of arbitrary isotropic lens functions that can be accommodated in a biaxial dielectric medium while maintaining symmetry. Because of this maximum functionality, the method may help to alleviate some problems traditionally associated with the use of transformation optics in dielectric media (no wasted polarizations) and aid future existence proofs on the possibility or impossibility of certain lens types.

Based on the method described, a Janus device having two different lens functions for unpolarized light along equatorial and polar plane "faces" was designed. The developed method of making use of biaxiality in dielectrics may lead to other interesting and useful optical devices in the future.

1. Leonhardt, U. \& Philbin, T. G. Geometry and Light: The Science of Invisibility (Dover, New York, 2010)

2. Danner, A. J., Tyc, T. \& Leonhardt, U. Controlling birefringence in dielectrics. Nat. Photon. 5, 357-359 (2011)

3. Maxwell, J. C. Camb. Dublin Math. J. 8, 188 (1854).

4. Eaton, J. E. On spherically symmetric lenses. Trans. IRE Antennas Propag. 4, 66-71 (1952).

5. Luneburg, R. K. Mathematical Theory of Optics, 2nd ed (University of California Press, Berkeley, CA, 1964).

6. Pendry, J. B., Schurig, D. \& Smith, D. R. Controlling electromagnetic fields. Science 312, 1780-1782 (2006).

7. Miñano, J. C. Perfect imaging in a homogeneous three dimensional region. Opt. Express 14, 9627-9635 (2006).

8. Born, M. \& Wolf, E. Principles of Optics (Cambridge University Press, Cambridge, 1999).

9. Akbarzadeh, A. \& Danner, A. J. Generalization of ray tracing in a linear inhomogeneous anisotropic medium: a coordinate-free approach. J. Opt. Soc. Am. A 27, 2558-2562 (2010).
10. Smolyaninova, V. N., Ermer, H. K., Piazza, A., Schaefer, D. \& Smolyaninov, I. I. Experimental demonstration of birefringent transformation optics devices. Phys. Rev. B 87, 075406 (2013)

11. Li, J. \& Pendry, J. B. Hiding under the carpet: a new strategy for cloaking. Phys. Rev. Lett. 101, 203901 (2008)

12. Shalaev, V. M. Optical negative-index metamaterials. Nat. Photon. 1, 41-48 (2007).

13. Boltasseva, A. \& Shalaev, V. M. Fabrication of optical negative-index metamaterials: recent advances and outlook. Metamaterials 2, 1-17 (2008).

14. Hashemi, H., Zhang, B., Joannopoulos, J. D. \& Johnson, S. G. Delay-bandwidth and delay-loss limitations for cloaking of large objects. Phys. Rev. Lett. 104, 253903 (2010).

15. Leonhardt, U. Optical conformal mapping. Science 312, 1777-1780 (2006).

16. Zentgraf, T., Valentine, J., Tapia, N., Li, J. \& Zhang, X. An optical “Janus” device for integrated photonics. Adv. Mater. 22, 2561-2564 (2010).

17. Hamilton, W. R. Third supplement to an essay on the theory of systems of rays. Trans. Royal Irish Acad. 17, 1-144 (1837).

18. Berry, M. V. \& Jeffrey, M. R. Conical diffraction: Hamilton's diabolical point at the heart of crystal optics. Prog. Opt. 50, 13-50 (2007).

19. Landau, L. D. \& Lifshits, E. M. A Shorter Course of Theoretical Physics, 1st ed (Pergamon Press, Oxford, 1972).

20. Hendi, A., Henn, J. \& Leonhardt, U. Ambiguities in the scattering tomography for central potentials. Phys. Rev. Lett. 97, 073902 (2006).

21. Danner, A. J. Singularity removal in optical instruments without reflections or induced birefringence. New J. Phys. 12, 113008 (2010)

\section{Acknowledgments}

The authors gratefully thank Tomáš Tyc for his helpful discussions and motivation during the early stages of this work. The authors also acknowledge support from grant No. R-263-000-690-112 of Singapore's Ministry of Education Tier 1 Academic Research Fund.

\section{Author contributions}

AA and AJD wrote the main manuscript text; AA prepared all figures. QCW contributed to the "Janus" device idea. All authors reviewed the manuscript.

\section{Additional information}

Supplementary information accompanies this paper at http://www.nature.com/ scientificreports

Competing financial interests: The authors declare no competing financial interests.

How to cite this article: Akbarzadeh, A., Qiu, C. \& Danner, A.J. Exploiting design freedom in biaxial dielectrics to enable spatially overlapping optical instruments. Sci. Rep. 3, 2055; DOI:10.1038/srep02055 (2013)

This work is licensed under a Creative Commons Attribution-

NonCommercial-ShareAlike 3.0 Unported license. To view a copy of this license, visit http://creativecommons.org/licenses/by-nc-sa/3.0 